\title{
Saudi Gastroenterology Association Guidelines for the Diagnosis and Management of Hepatocellular Carcinoma: Summary of Recommendations
}

Guidelines Editors: Ayman A Abdo, Huda Al Abdul Karim, Turki Al Fuhaid, Faisal M Sanai, Munthir Kabbani, AbduIRahman Al Jumah, Kelly Burak

$\mathrm{R}$ ecognizing the high prevalence of hepatocellular carcinoma (HCC) in Saudi Arabia and the difficulties often faced in acTeferral of HCC patients, a committee was formed at King Khalid University Hospital (KKUH) in Riyadh (first three editors, AAA, HAK, TF) who wrote the first draft of these guidelines. These editors first performed a widely based literature search on all aspects of the epidemiology, natural history, risk factors, diagnosis, and management of HCC. The literature was examined critically and the available evidence was then classified according to its strength.

After approval and implementation of the initial guidelines at $\mathrm{KKUH}$, it was decided to use these guidelines as the backbone for a national project for Saudi Arabia. Three other editors (FMS, MK, AJ) from three of the major hospitals in Saudi Arabia updated the document. After that, representative expert contributors were invited to review the document. These contributors (listed at the end of this article), who are experts in different specialties related to HCC management from different institutions in Saudi Arabia, then further refined and updated the sections related to their expertise. Multiple meetings were then called to approve the final document. An international expert in the area of $\mathrm{HCC}(\mathrm{KB})$ then reviewed the document and offered useful suggestions and improvements. After that, the Saudi Gastroenterology Association formed a committee that reviewed these guidelines and approved them as the "Saudi Guidelines for the Diagnosis and Management of Hepatocellular Carcinoma". The recommendations are based on the best available evidence but were tailored to the patients treated in Saudi Arabia.

We have benefited from the two published European guidelines. The first is the conclusion of the Barcelona-2000 European Association for the Study of the Liver Conference, ${ }^{1}$ and the second is the guidelines published by the British Society of Gastroenterology. ${ }^{2}$ In addition, we have benefited from the special dedicated supplement issue of Gastroenterology (November 2004) containing extensive reviews of the topic and from the American Association for the Study of the Liver (AASLD) guidelines that were recently published. ${ }^{3} \mathrm{We}$ hope that these guidelines will improve care for patients with $\mathrm{HCC}$ and facilitate the performance of excellent research on this important medical problem.

The full guidelines, which are completely referenced and contain detailed recommendations, are available on the Annals of Saudi Medicine website (www.saudiannals.net). The full guidelines include a grading of recommendations based on the quality of the evidence for the rec-
Correspondence and reprint

requests:

Ayman A. Abdo, MD

College of Medicine

King Saud University

P.O. Box 2925(59)

Riyadh 11461

Saudi Arabia

Tel: +966-1-467 1215

Fax: +966-1-4671217

abdoayman@hotmail.com

Accepted for publication

June 2006

Ann Saudi Med 2006;26(4):261-265

For FuLL GUIDELINES Go To:

http://www.saudiannals.net/

SGAHCCguidelines/ 
ommendation. A summary of the guidelines is presented here.

\section{Epidemiology}

In Saudi Arabia, liver cancer accounts for $6.1 \%$ of all newly diagnosed cancers, according to the most recent cancer registry covering the years 1999-2000. ${ }^{4}$ $\mathrm{HCC}$ was the second most common cancer affecting Saudi males and the eighth most common cancer affecting females with an overall age standardized rate of 4.5/100 000 population. The male to female ratio is 279:100. In Saudi Arabia, hepatocellular carcinoma accounts for $87.6 \%$ of all liver cancers. The median age at diagnosis is 65 years for males and 60 years for females. This incidence of HCC in Saudi Arabia is not surprising given the high prevalence of the two major risk factors, namely hepatitis $B$ and hepatitis $\mathrm{C}$ infection. Patients with cirrhosis of any etiology, but especially with cirrhosis caused by hepatitis B or $\mathrm{C}$ are at high risk for the development of $\mathrm{HCC}$ and these patients should be the target of a screening program. Large studies are needed to further define the epidemiologic features of HCC in Saudi Arabia, but clearly the problem is large enough that more resources should be allocated to it.

\section{Clinical presentation}

HCC usually presents with weight loss and right upper quadrant pain in addition to the symptoms and signs of underlying liver cirrhosis (weakness, abdominal swelling, non-specific gastrointestinal symptoms and jaundice), but physical findings will vary with the stage of the disease. If the tumor is small, no signs may be found except those related to the underlying cirrhosis. In more advanced cases, hepatomegaly is common, with the possibility of palpating a mass or a hard irregular liver surface which in turn may be tender. Special clinical scenarios that should raise the suspicion of $\mathrm{HCC}$ include acute deterioration of liver function in a patient with stable cirrhosis, new onset ascites, and acute intraabdominal bleeding. Investigations for HCC should be initiated in patients with stable cirrhosis if there is rapid deterioration of liver function, new onset ascites, weight loss, or increased jaundice.

\section{Diagnosis}

Ultrasonography (US) should be the initial radiological investigation when $\mathrm{HCC}$ is suspected. Further imaging should be performed if an abnormality is found or if the suspicion of HCC is high even if the US is normal. A triphasic CT scan or MRI are

\section{The goals of these guidelines}

- To provide an evidence-based review of the diagnosis and management of hepatocellular carcinoma (HCC).

- To help initiate plans to prevent HCC.

- To enhance early and accurate diagnosis of patients with HCC.

- To provide a suggested evidence-based approach for the management of HCC patients.

- To facilitate a more effective referral system between primary/ secondary care physicians and tertiary care centers where advanced treatments are available.

- To help adopt a more effective triaging system of patients within tertiary care centers.

- To create a complementary system between the major tertiary care centers in which specific centers may specialize in certain aspects of management or care.

- To identify research questions that are applicable to our local circumstances and resources and to facilitate recruiting patients in these studies.

the radiological procedures of choice to confirm the diagnosis of HCC. It is extremely important that the CT scan is done by a standard triphasic technique and is read by a trained abdominal radiologist.

The diagnosis of HCC may be positively made if the liver is cirrhotic, the lesion is larger than $2 \mathrm{~cm}$ in diameter, and either the alpha-fetoprotein (AFP) is higher than $400 \mathrm{ng} / \mathrm{L}$ and one imaging modality confirms early arterial enhancement and venous washout, or the AFP is normal and two imaging modalities confirm early arterial enhancement and venous washout. Although AFP is a poor screening tool for HCC it should be measured in all suspected cases. Levels below $400 \mathrm{ng} / \mathrm{L}$ may be seen in patients with active hepatitis without HCC. Levels above $1000 \mathrm{ng} / \mathrm{L}$ are more specific for HCC. A consistently rising AFP is highly suggestive of HCC.

A histological diagnosis is recommended only if a lesion is found in a non-cirrhotic liver and radiological investigations are not conclusive for hemangio$\mathrm{ma}$, focal nodular hyperplasia, or adenoma, or if the suspicion of HCC is high but the non-invasive criteria (mentioned above) are not satisfied. Otherwise, histological confirmation is not required.

\section{Evaluation and Staging}

Liver tumor weekly rounds should be started at every tertiary care center dealing with HCC patients. In this one-hour meeting, all cases of liver tumors should be discussed. The group should be composed 
of a hepatologist, an oncologist, a hepatobiliary surgeon, and an interventional radiologist. The goal of the meeting is to discuss new cases of liver tumors and to reach a joint decision on the most appropriate management route for these patients. Weekly rounds should also improve recruitment in clinical trials and should serve in teaching residents and fellows.

Patients with a liver mass should be evaluated in the weekly liver tumor rounds. This evaluation will help determine the next step in obtaining a final diagnosis, determine the extent of hepatic involvement, rule out extra-hepatic disease, evaluate the general medical status and performance of the patient, and evaluate functional hepatic reserve.

Initial evaluation of patients with HCC should include a complete history, a full physical examination, initial laboratory tests including a complete blood count, random serum glucose, serum electrolytes, renal function, alpha fetoprotein, serum calcium, prothrombin time, and liver function tests, a chest $\mathrm{x}$ - ray (or a CT of the chest if there are suspicious lesions for metastases on the chest X-ray), and a bone scan (on initial visit only and every 9 months thereafter). Follow-up visits should also include all of the above at least every 3 to 6 months as long as the patient is in an active treatment arm of the management plan. Although each staging system has its own advantages and disadvantages, the CLIP (Cancer of the Liver Italian Program) score enjoys epidemiologic support and prospective validation while the BCLC (Barcelona Clinic Liver Cancer Classification System) is probably the best in planning future treatment plans. See the full guidelines for more information.

\section{Management}

Patients with HCC should be managed in tertiary care centers where expertise is available. The management plan for patients with HCC should be constructed in a multi-disciplinary forum consisting of a hepatologist, oncologist, interventional radiologist, and a hepatobiliary surgeon. The decision on the best treatment modality should be based on the number of lesions, the size of lesions, the status of the underlying liver, the status of the portal vein, the performance status of the patient, the local expertise, and patient preferences (Figures 1-4).

Indications for liver transplantation are: liver cirrhosis, a single lesion less than $5 \mathrm{~cm}$ or less than 3 lesions smaller than $3 \mathrm{~cm}$ each, no evidence of portal vein invasion or extra-hepatic spread, and no contraindications for liver transplantation. To prevent the patient from outgrowing these transplantation criteria while waiting on the transplant list, local ablative therapy or chemoembolization may be considered to control tumor growth. The utility of cadaveric liver transplantation in the management of $\mathrm{HCC}$ in Saudi Arabia is limited due to the relative lack of donors leading to long waiting list time. Living related transplantation should be explored in future studies in Saudi Arabia as an alternative for long waiting lists of cadaveric transplantation.

Indications for partial liver resection are: no cirrhosis or early cirrhosis with normal bilirubin and no signs of clinically relevant portal hypertension (defined as presence of varices, splenomegaly, platelet count $<100000$ or a hepatic vein pressure gradient $>10 \mathrm{~mm} \mathrm{Hg}$ ), no evidence of portal vein or its main branches invasion or extrahepatic spread, and a tumor that is technically resectable.

Indications for local ablative therapy are: the presence of three lesions or less that are smaller than 4 $\mathrm{cm}$ in diameter, no evidence of extra- hepatic spread, and a patient who is not a candidate for liver resection. The local ablative procedure of choice is radiofrequency ablation (RFA). RFA is more effective than alcohol injection (especially in lesions larger than $3 \mathrm{~cm}$ ) but with a slightly higher risk. It may be associated with improved survival. Technical considerations in regards to the site of the lesion may favor one method over the other.

Indications of chemoembolization are: a single or multiple lesions larger than $4 \mathrm{~cm}$ and smaller than 10 $\mathrm{cm}$, no evidence of advanced cirrhosis (Child class A or B, according to the Child-Pugh classification for assessment of liver function), and a patent portal vein with normal flow. Chemoembolization should be performed at baseline, 2 months, 6 months, and every 6 months thereafter for 6 cycles as long as the patient is responding.

Patients with vascular invasion, extra-hepatic spread, or diffuse multifocal disease should receive palliative care only and may be enrolled in trials of systemic chemotherapy. Octreotide cannot be recommended based on available evidence. Patients with Child-Pugh class $\mathrm{C}$ cirrhosis should be offered medical treatment only. A specialized palliative care team should be involved in the management of endstage HCC.

\section{Prevention}

The vaccination of all children in Saudi Arabia against hepatitis B starting at birth should be maintained and further encouraged. Vaccination of people 

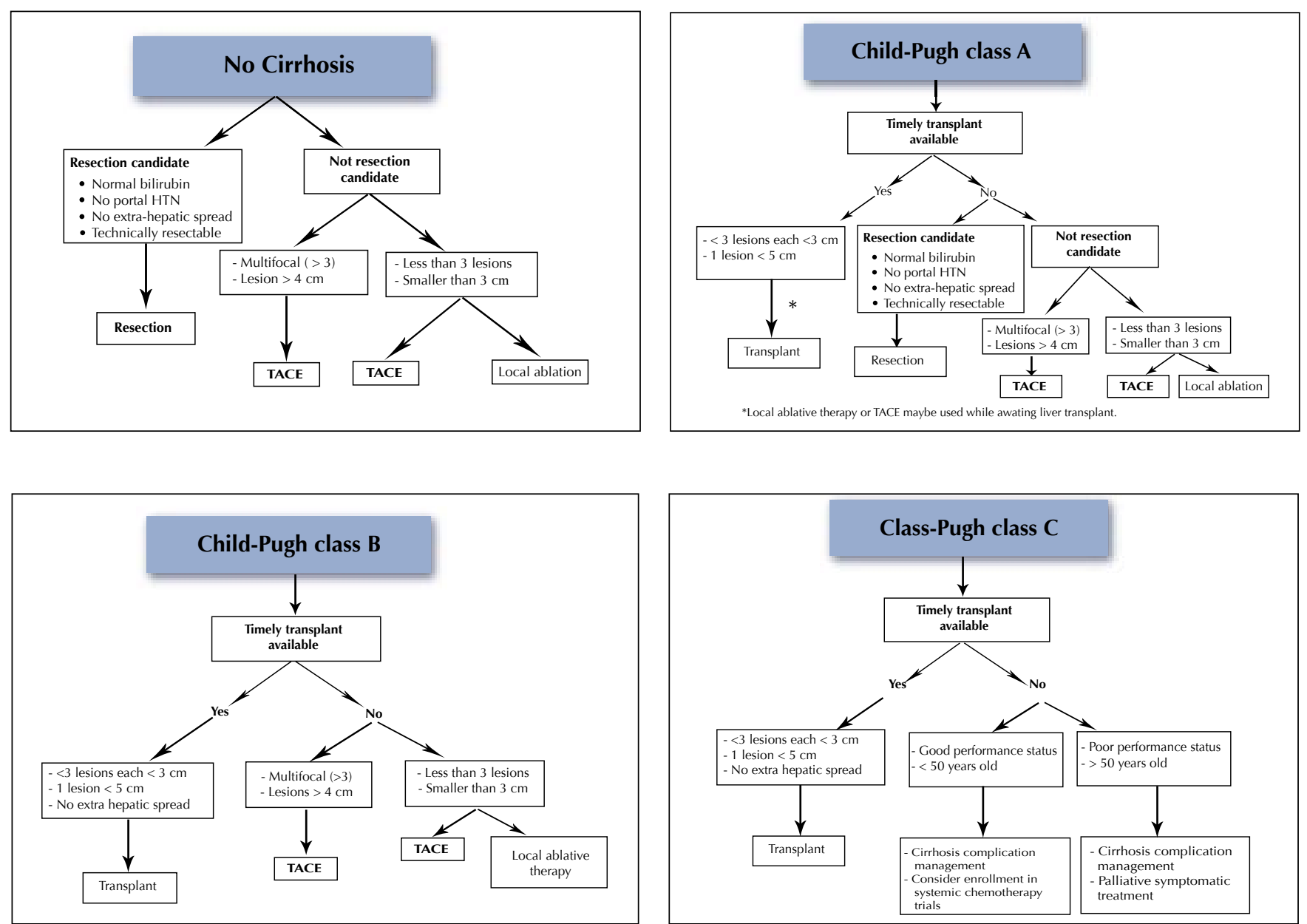

Figure 1. Management algorithms for hepatocellular carcinoma with no cirrhosis and Child-Pugh classes $A, B$, and $C$ cirrhosis (TACE: transarterial chemoembolization, HTN: hypertension)

at risk for hepatitis $B$ infection should be encouraged. Post-exposure prophylaxis for hepatitis B should be implemented in all hospitals. Post-exposure testing for hepatitis $\mathrm{C}$ using a PCR-based test and early treatment of hepatitis $\mathrm{C}$ should be implemented. Treatment with a pegylated interferon plus ribavirin should be considered for all patients with hepatitis $\mathrm{C}$ who have elevated liver enzymes and active hepatitis on liver biopsy and a positive HCV-RNA test. All patients with chronic hepatitis $B$, elevated liver enzymes, a liver biopsy showing active inflammation or significant fibrosis, and an HBV DNA more than $10^{5}$ copies $/ \mathrm{mL}$ should be considered for antiviral therapy using pegylated interferon monotherapy or antiviral therapy using lamivudine, adefovir or entecavir.

All patients with hepatitis B-related end-stage liver cirrhosis should be considered for long term lamivudine/adefovir therapy as this is expected to reduce their mortality and reduce the incidence of HCC. Screening using AFP and US should be implemented in all cirrhotic patients every 6 months. Screening of patients with chronic hepatitis B without evidence of cirrhosis cannot be recommended at this time but may be offered in certain high-risk groups like patients older than 40 years of age and patients with a family history of HCC. There is no evidence to recommend screening of patients with chronic hepatitis $\mathrm{C}$ without cirrhosis. Any patient with a positive US should undergo further imaging with a triphasic CT scan or an MRI.

\section{Research needs}

Large epidemiologic studies identifying patterns of disease and clinico-pathological aspects of this disease are needed in Saudi Arabia. Also needed is a large study to assess the feasibility, effectiveness, and 
cost-effectiveness of a screening program in patients with cirrhosis secondary to hepatitis B or hepatitis $\mathrm{C}$, and in patients with chronic hepatitis $\mathrm{B}$ without cirrhosis. The arms of the treatment algorithm need to be further explored by well designed, preferably randomized studies comparing resection with local ablative therapy or transarterial chemoembolization (TACE) in patients with no or early cirrhosis and lesions less than $5 \mathrm{~cm}$, comparing different modalities of local ablative therapies, comparing local ablative therapy vs. TACE in lesions less than $5 \mathrm{~cm}$, examining the role of TACE in shrinking tumors in preparation for resection or liver transplantation, and assessing the utility of new systemic chemotherapeutic agents in nonresectable tumors versus standard medical therapy.

\section{Guidelines Contributors}

King Khalid University Hospital, Riyadh:

Ayman Abdo (coordinator), Huda Al Abdulkareem, Nizar Al Nakshabandi, Turki Al Fubaid, Wael Al Kattan, Abmed Al Sagheir, Saleb Al Sumayer

King Faisal Specialist Hospital and Research Center, Riyadh:

Monthir Kabbani (coordinator), Shawki Bazerbashi, Hamad Al Ashgar, Mohammad Al Sagheir, Mohammad Al Sofayan, Hamad Al Subaibani, Mohammad Al Shammari

Riyadh Armed Forces Hospital:

Faisal Sanai (coordinator), Khalid Bzeizi, Molfi Al Otaibi

King Fabad Medical City:

Ali Al Shanqeety

King Abdul Aziz Medical City:

Abdulrabman Al Jumab (coordinator), AbdulJaleel Al Alwan

King Abdul Aziz University Hospital, Jeddah:

Hisham Akbar

King Fahad Hospital-Jeddah

Mohamed Babatin, Abdallah Al-Ghamdi

King Faisal University Hospital, Al Khobar:

Mona Ismael

King Khalid University, Abha:

Sulaiman Al Humaid

International expert:

Kelly Burak, University of Calgary, Canada

\section{References}

1. Bruix J, Sherman M, Llovet JM, Beaugrand M, Lencioni $M$, Burroughs AK et al. Clinical management of hepatocellular carcinoma. Conclusions of the Barcelona-2000 EASL Conference. J Hepatol
2001; 35:421-430

2. Ryder S. Guidlines for the diagnosis and treatment of hepatocellular carcinoma in adults. Gut 2003; 52:S3-1-S3-8.
3. Bruix J, Sherman M. Management of hepatocellular carcinoma. Hepatology 2005; 42(5):1208-1236. 4. Kingdom of Saudi Arabia Ministry Of Health, $\mathrm{Na}$ tional Cancer Registry (1997-1998). 2001. 